\title{
Recyclable Calix[4]arene-Lanthanoid Luminescent Hybrid Materials with Color-Tuning and Color-Switching Properties
}

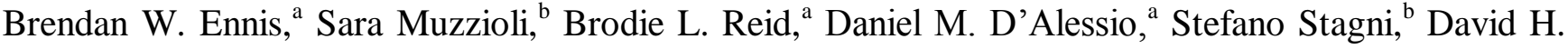 \\ Brown, ${ }^{\mathrm{a}, *}$ Mark I. Ogden, ${ }^{\mathrm{a}, *}$ Massimiliano Massi ${ }^{\mathrm{a}, *}$ \\ ${ }^{a}$ Department of Chemistry, Curtin University, Kent Street, Bentley WA 6102, Australia. \\ ${ }^{\mathrm{b}}$ Department of Physical and Inorganic Chemistry, University of Bologna, viale del Risorgimento 4, \\ 40126 Bologna, Italy.
}

\begin{abstract}
Inorganic/organic hybrid materials combine the properties of both components providing functionality with a wide range of potential applications. Phase segregation of the inorganic and organic components is a common challenge in these systems, which is overcome here by copolymerizing a metal-free calixarene ionophore and methyl methacrylate. A lanthanoid ion is then added using a swelling-deswelling procedure. The resulting luminescent hybrid materials can be made to emit any required color, including white light, by loading with an appropriate mixture of lanthanoids. The gradation of the emitted color can also be finely adjusted by changing the excitation wavelength. The polymer monolith can be recycled to emit a different color by swelling with a solution containing a different metal ion. This methodology is flexible and has the potential to be extended to many different ionophores and polymer matrices.
\end{abstract}

\section{Introduction}

Hybrid materials combine the functional properties of the guest with the mechanical and thermal properties of the host. ${ }^{1,2}$ In the case of inorganic/organic hybrid materials, a metal-based coordination complex is generally included into a polymeric matrix. ${ }^{3}$ This strategy has enabled the preparation of a vast library of hybrid materials where the magnetic, optical, and/or catalytic functionalities of the metal host are added to stable and readily processable organic polymers. The rapidly growing research activity in hybrid materials is driven, at least in part, by the fact that these materials have potential applications in a variety of areas such as magnetic memories, optical displays, light sources, telecommunication devices, lasers, agriculture, sensors, medical diagnostics, and metal extraction. ${ }^{1,4-9}$

Lanthanoid-based hybrid materials have functions that are associated with the specific properties of the 
4f electrons. ${ }^{1,5}$ In particular, this class of materials has been widely exploited for the optical properties of the lanthanoid elements, whose emission spans from the visible to the infrared range. ${ }^{5,10}$ Lanthanoid elements need to be excited by light-absorbing chromophores, a phenomenon known as the antenna ef$f e c t,{ }^{11}$ as the direct excitation of the lanthanoid ions is inefficient due to $f$ - $f$ electronic transitions being quantum-mechanically forbidden by symmetry and often by spin selection rules. The advantage of using lanthanoid elements as light emitters resides in their purity of colors, which is almost completely unaffected by their chemical environment due to the inner core nature of the $4 f$ orbitals. ${ }^{12}$ Moreover, the combination of different lanthanoid elements can also be used to achieve white light emission, which has been exploited in the preparation of a variety of advanced materials including emissive glasses, phosphors, and metal-organic frameworks (MOFs). ${ }^{13-18}$

A common issue associated with the preparation of lanthanoid-based metallopolymers is phase segregation, where the metal complex aggregates in a separated phase from the rest of the polymeric matrix. ${ }^{1,3}$, 19 This phenomenon leads to unwanted characteristics of the final material such as loss of transparency and non-homogeneous emission. Therefore, the maximum amount of metal complex that can be loaded into the polymeric matrix, either by simply blending or covalently grafting, is limited to the solubility of the complex in the monomer itself, when the polymerisation is performed neat, or in the organic solvent, when polymerisation is performed in solution.

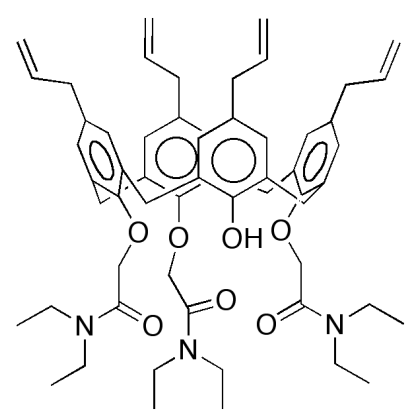

Figure 1. Calix[4]arene ligand $\mathbf{1 H}$ used for the preparation of the crosslinked PMMA hybrid materials.

We have recently demonstrated how phase segregation issues can be readily avoided by copolymerizing a highly soluble multidentate ligand, such as the calix[4]arene $1 \mathrm{H}$ (Figure 1), with a suitable monomer and then swelling the resulting crosslinked polymer with a solution containing the lanthanoid of choice. ${ }^{20}$ With this approach the maximum complex loading is determined by the solubility of the ligand instead of the solubility of the complex, and as such a high concentration of inorganic dopants can be achieved without loss of transparency or phase segregation. Extending our investigation using poly(methyl methacrylate) (PMMA) as the transparent polymeric host, we now report how this method- 
ology can be exploited for mixture of lanthanoid ions, thus obtaining variations of colors, and also for the preparation of recyclable hybrid materials, whose optical properties can be readily switched by exchanging the lanthanoid of choice without altering the chemical and structural properties of the hybrid material. Moreover, we demonstrate how mixtures of lanthanoid elements can be loaded within the material to finely tune color variations, and even obtain white light emission, as illustrated in Figure 2. Throughout the manuscript, we are going to use CIE-xy chromaticity diagrams to estimated the emitted colors from the materials, therefore pure white light will be represented by $(x=0.333, y=0.333)$.

Results and Discussion

\section{Preparation of the luminescent materials by polymer loading}

The blank cross-linked PMMA materials were prepared by neat radical polymerisation. Lauroyl peroxide ( $0.025 \%$ mol. eq.), ethylene glycol dimethacrylate $(0.3 \%$ mol. eq. $)$, calix[4]arene $1 \mathrm{H}(0.2 \%$ mol. eq. $)$ were added to methyl methacrylate and the solution was heated inside a glass tube. The polymerisation was initiated at $80^{\circ} \mathrm{C}$ for 3 minutes, followed by rapid cooling in ice water. The solution was then gradually heated from 60 to $100{ }^{\circ} \mathrm{C}$ for about 3 days. This temperature profile ensures the polymeric matrices are uniform, transparent as well as free of cracks and bubbles (Figure 2). ${ }^{21}$

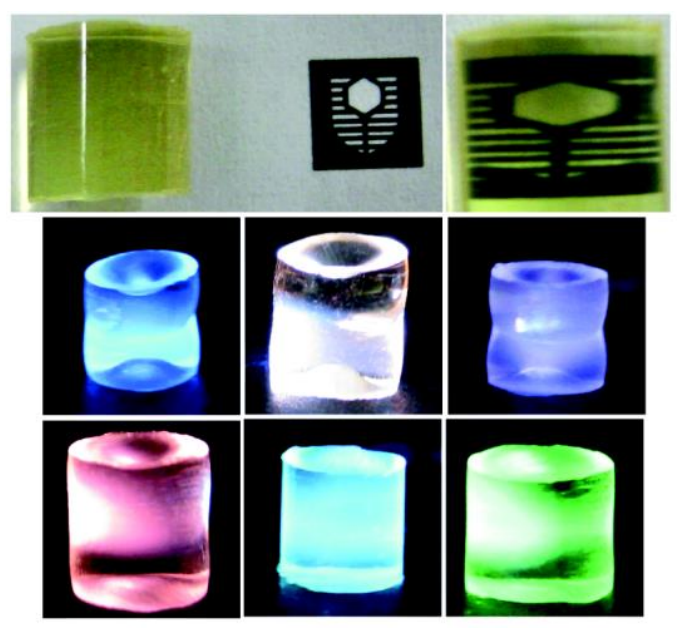

Figure 2. Examples of lanthanoid metallopolymers demonstrating the transparency of the matrix (top) and variations of colors obtained by loading single lanthanoid salts or mixtures. All the monoliths in the Figure have been excited at $\lambda_{\mathrm{ex}}=330 \mathrm{~nm}$.

The loading of the blank materials was performed by swelling the monoliths in a 1:1 dichloro- 
methane/ethanol mixture containing the desired lanthanoid salts at a concentration of ca. $10^{-3} \mathrm{M}^{20}$ The volume of solution used corresponded to $\sim 5$ eq. of lanthanoid ions with respect to the amount of calix[4]arene ligand incorporated within the monolith. A swelling of about $150 \pm 5 \%$ of the original volume occurs when the monoliths are immersed in the mixture of solvents, indicating efficient cross-linking of the polymeric chains. ${ }^{3,21}$ After the loading has occurred, the excess metal cations are then removed by immersing the monoliths in fresh metal-free solvent mixtures. Eventually, the monoliths are de-swollen in air, returning to their original volume and shape (deformation of the monoliths occurred if de-swelling was accelerated under reduced pressure). Typically, DMSO-solvated lanthanoid nitrate salts $\left[\mathrm{Ln}\left(\mathrm{NO}_{3}\right)_{3}(\mathrm{DMSO})_{\mathrm{n}}\right] \mathrm{n}=4-6$ were used, instead of the hydrated salts, to increase their solubility in the organic mixture and to reduce the quenching effects from the lanthanoid ions via multiphonon relaxation caused by coordinated water molecules. ${ }^{11,20}$ By comparing with previously obtained crystal structures of complexes formed by $1 \mathrm{H}$ and $\mathrm{Tb}$ ions, it would be expected that one molecules of water coordinates to the metal centre. ${ }^{20}$

Color emission from mixtures of $\mathrm{Sm}, \mathrm{Tb}$ and $\mathrm{Tm}$ with $1 \mathrm{H}$ in solution

Our first investigation focused on mixing the different lanthanoid cations in solution at various molar ratios to estimate the effect on the emitted color. In general, white light is obtained through a balanced combination of the red, green, and blue colors. In accordance with these colors, the lanthanoid elements of choice are $\mathrm{Sm}, \mathrm{Tb}$, and Tm: these are well known orange, green, and blue emitters via their ${ }^{6} H_{J} \leftarrow{ }^{4} G_{5 / 2},{ }^{7} F_{J} \leftarrow{ }^{5} D_{4}$, and ${ }^{3} H_{J} \leftarrow{ }^{1} G_{4}$ phosphorescent transitions, respectively. ${ }^{11}$ Sm was chosen instead of $\mathrm{Eu}$ as calix[4]arene complexes of the latter are known to undergo quenching of the ${ }^{5} D_{0}$ excited state via a charge transfer with formation of divalent $\mathrm{Eu}$ and oxidation of the phenolic ligand. ${ }^{22}$ The color mixing effect was directly evident from qualitative solution studies, where a variable mixture of Sm, Tb, and Tm salts were added to a $10^{-3} \mathrm{M}$ solution of $1 \mathrm{H}$ in $1: 1$ dichloromethane/ethanol, as the corresponding complexes have a relatively high solubility in this mixture. Upon excitation at $\lambda_{\mathrm{ex}}=330 \mathrm{~nm}$, the solutions were characterized by a uniform color obtained by the combined emission of the three lanthanoid cations (Figure 3). The closest emission to white light was obtained by combining a mixture of lanthanoid salts with a molar ratio of 8:9:91 $\mathrm{Sm} / \mathrm{Tb} / \mathrm{Tm}$. The higher concentration of Tm required in the mixture was ascribed to a lower quantum yield for the $[\operatorname{Tm}(\mathbf{1 H})]^{3+}$ complex. In fact, the quantum yields of the complexes were estimated of the order of $3 \%$ for $\mathrm{Tb}, 2 \%$ for $\mathrm{Sm}$, and below $0.3 \%$ for $\mathrm{Tm}$. The relatively low quantum yields in solution could be ascribed to a combination of factors such as non-optimized energy difference between the sensitizer excited state and the Ln accepting state, as well as quenching promoted by the phenolic $\mathrm{OH}$ and coordinated ethanol molecules. ${ }^{23}$ 


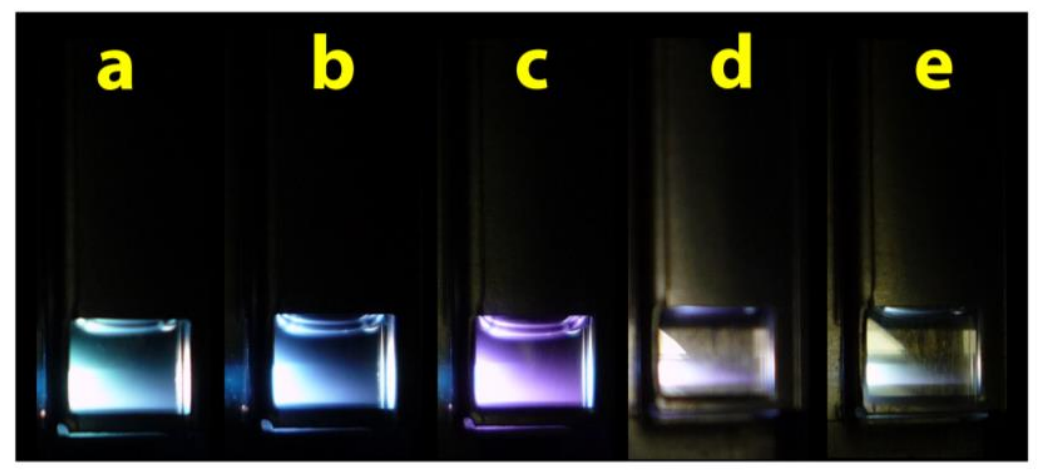

Figure 3. Emission from dichloromethane/ethanol solutions containing $1 \mathrm{H}$ and a mixture of $\mathrm{Sm} / \mathrm{Tb} / \mathrm{Tm}$ of the following ratios: a) 5:2:93; b) 5:1:94; c) 6:1:93; d) 7:1:92; e) 8:9:91.

Photophysical properties of loaded polymers with mixtures of lanthanoid ions

When the blank polymers were loaded with the mixtures of the three lanthanoid ions found in solution with the unpolymerized $1 \mathrm{H}$ complexes, the emitted colors from the materials did not correspond to the ones observed from the photoluminescence of the solutions. Generally a molar excess of lanthanoid ions with respect to the total molar amount of polymerized calixarene was used to favor quantitate complexation. Uncomplexed ions were then removed by washing, but in any case we have previously demonstrated that lanthanoid ions present in the polymer, but not coordinated to the calixarene, do not contribute to the emission due to lack of sensitisation. ${ }^{20}$ Although uniformly mixed colors were detected from the PMMA monoliths, the balance of the three individual colors was altered. On analyzing the emission profiles from the hybrid materials, a broad band could be highlighted in the blue region (400-500 nm), superimposed on the ${ }^{7} F_{6} \leftarrow{ }^{5} D_{4}$ and ${ }^{3} H_{6} \leftarrow{ }^{1} G_{5 / 2}$ transitions of $\mathrm{Tb}$ and Tm, respectively (Figure 4). The same broad band is also visible in the emission from the monolith loaded exclusively with Tm and, to a lesser extent, Sm (Figure 5). The broad nature of this new band was attributed to the superimposition of phosphorescent emission from the ${ }^{3}\left(\pi \pi^{*}\right)$ state of $\mathbf{1 H}$ and fluorescent emission from the solid polymeric matrix. To confirm this conclusion we analyzed the emission and excitation spectra of a blank unloaded monolith and a monolith crosslinked without the calixarene ligand, both of which present identical profiles. Furthermore, the emission and excitation spectra of a monolith loaded exclusively with $\left[\mathrm{Gd}\left(\mathrm{NO}_{3}\right)_{3}(\mathrm{DMSO})_{\mathrm{n}}\right]$ was evaluated. These spectra are shown in Figure 5. By analyzing the spectra it appears that there is negligible contribution in emission from calixarene ligands that have not been complexes by a lanthanoid cation. In this case the broad blue band seems to originate from fluorescent emission from the matrix, which is further confirmed by its correspondingly short excited state lifetime fitted with a biexponential contribution of $1.6 \mathrm{~ns}(50 \%)$ and $6.0 \mathrm{~ns}(50 \%)$. The fluorescent emis- 
sion of the unloaded monolith presents a maximum in the excitation spectrum around $380 \mathrm{~nm}$, further supporting the fact that the major contributor is the matrix rather than the calixarene, since the latter generally presents absorption and excitation maxima centered around $330 \mathrm{~nm}$. On the other hand, the monolith loaded with Gd ions presents a similar blue emission, although slightly narrower than the previous case. This emission was ascribed to decay from the ${ }^{3}\left(\pi \pi^{*}\right)$ excited state of the calixarene, as suggested by its loner excited state decay of ca. $10 \mu \mathrm{s}$. However, the excitation spectrum reveals some contribution from the fluorescence of the matrix with a shoulder appearing around $380 \mathrm{~nm}$. The same conclusion can be drawn by analyzing the excitation spectrum of the material loaded with Tm, where two distinct maxima appear when monitoring the emission intensity of the band at $470 \mathrm{~nm}$. The higher energy band around $330 \mathrm{~nm}$ corresponds to the excitation spectrum of the monolith loaded with $\mathrm{Sm}$, and $\mathrm{Tb}$ (Figure 5), thus indicating energy transfer from the ${ }^{3}\left(\pi \pi^{*}\right)$ excited state of the ligand. On the other hand, the lower energy band around $380 \mathrm{~nm}$ corresponds to the excitation profile of the unloaded blank material, suggesting the contribution comes from the fluorescence of the polymeric matrix. 


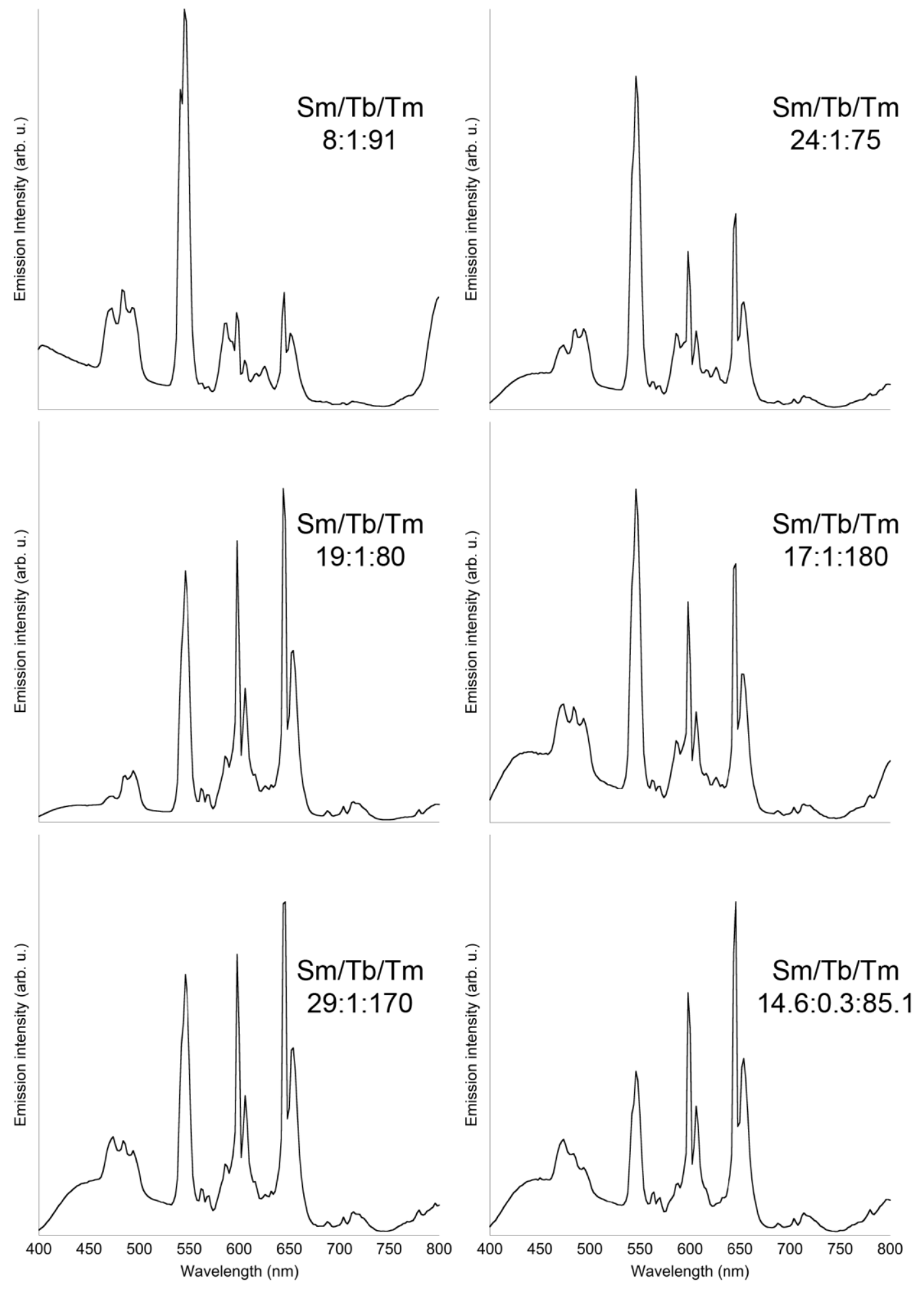

Figure 4. Emission profiles obtained from monolith loaded with variable mixtures of $\mathrm{Sm} / \mathrm{Tb} / \mathrm{Tm}$. All the emission spectra have been obtained with $\lambda_{\mathrm{ex}}=330 \mathrm{~nm}$. 

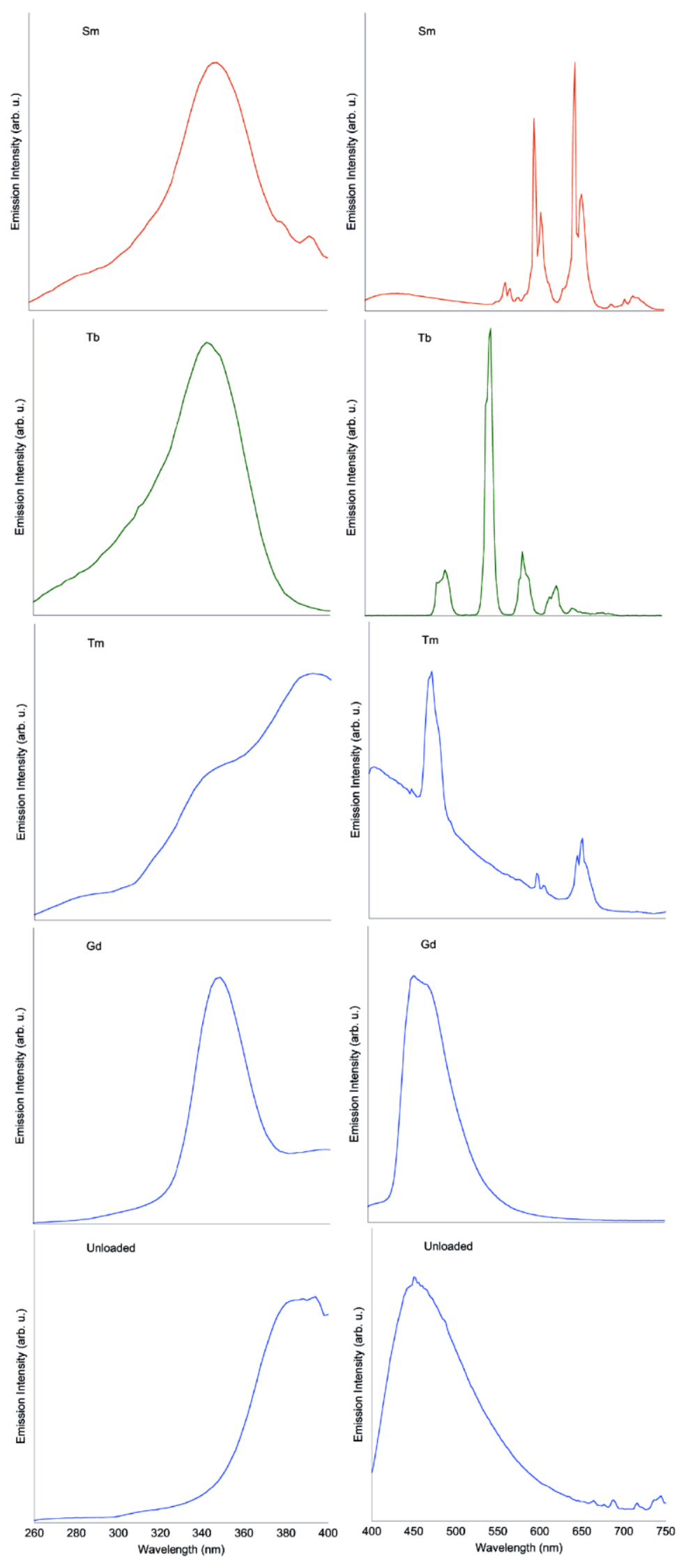

Figure 5. Excitation and emission profiles for a blank unloaded monolith and monolith loaded with a single lanthanoid cation. Each monolith is crosslinked with $0.2 \%$ molar equivalents with respect to the 
initial amount of methyl methacrylate. Each emission spectrum is obtained with $\lambda_{\mathrm{ex}}=330 \mathrm{~nm}$, aside from the unloaded monolith that was excited at $\lambda_{\mathrm{ex}}=370 \mathrm{~nm}$ Note that the emission and excitation spectra from the unloaded monoliths are identical to those of monoliths crosslinked in the absence of calixarene. The excitation spectra were recorded by following the intensity of the maximum in the emission spectrum: i) Sm $646 \mathrm{~nm}$; ii) Tb $546 \mathrm{~nm}$; iii) Tm $470 \mathrm{~nm}$; iv) Gd and unloaded $456 \mathrm{~nm}$.

A series of hybrid materials loaded with different molar ratios of the three lanthanoid salts was prepared (Figure 4). The resulting materials were characterized by homogeneous emission of colors with different hues, depending on the relative ratio of lanthanoid elements. The calculated CIE-xy coordinates for each of the different monoliths are reported in Figure 6 and Table 1. Balanced white light emission was eventually obtained using a 17:1:180 Sm/Tb/Tm molar ratio, to balance out for the increased emission in the blue region compared to the solution studies, where white light was obtained with a molar ratio of approximately 8:9:91 $\mathrm{Sm} / \mathrm{Tb} / \mathrm{Tm}$. The excited state lifetime decays $(\tau)$ measured at $\lambda_{\mathrm{em}}=546$, 598, and $646 \mathrm{~nm}$ (Table 2a) are comparable with the values obtained from monoliths loaded with either $\mathrm{Sm}$ or Tb (Table $2 \mathrm{~b}$ ). The $\tau$ data were satisfactorily fitted in all cases with biexponential decays, with good correspondance to the one measured from monoliths loaded with individual lanthanoids. The minimal variation between the excited state lifetime values in the white-emitting material and the monoliths loaded exclusively with one lanthanoid element suggests that energy transfer between the various lanthanoid complexes is not a dominant factor in the photophysical properties, at least with a loading of lanthanoid complex corresponding to ca. $0.2 \%$ molar equivalents. The lifetime decays in the $400-500 \mathrm{~nm}$ region were more complex and could not always be satisfactorily fitted with exponential functions. This was attributed to the presence of decays from multiple excited states of short and long lifetime such as i) the polymeric matrix [1.6 $\mathrm{ns}(50 \%)$ and $6.0 \mathrm{~ns}(50 \%)$ at $\lambda_{\mathrm{em}}=454 \mathrm{~nm}$ measured for the unloaded blank polymer]; ii) the ${ }^{3}\left(\pi \pi^{*}\right)$ of $1 \mathrm{H}$ [10 $\mu$ s at $\lambda_{\mathrm{em}}=456 \mathrm{~nm}$ measured for the polymer loaded with $\left.\mathrm{Gd}\right]$; iii) the $\mathrm{Tm}{ }^{3} H_{6} \leftarrow{ }^{1} G_{5 / 2}$ transition [3.51 ns $(74 \%)$ and $142.77 \mathrm{~ns}(26 \%)$ at $\lambda_{\mathrm{em}}=470 \mathrm{~nm}$ measured for the polymer loaded with Tm - it should be however noted that this Tm decay is mixed with the decay from the polymeric matrix]. 


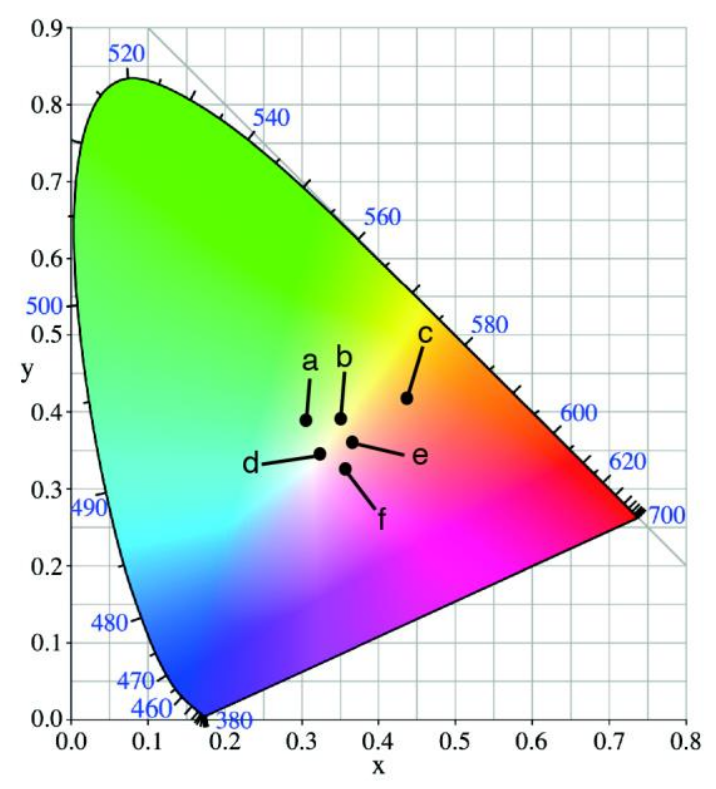

Figure 6. CIE-xy coordinates for the monoliths loaded with mixtures of $\mathrm{Sm} / \mathrm{Tb} / \mathrm{Tm}$ at different ratios: a) $8: 1: 91$; b) $24: 1: 75$; c) $19: 1: 80$; d) $17: 1: 180$; e) $29: 1: 170$; f) 14.6:0.3:85.1. The monoliths have been excited at $\lambda_{\mathrm{ex}}=330 \mathrm{~nm}$.

Table 1. CIE-xy coordinate $\mathrm{x}$ and $\mathrm{y}$ values for the monoliths loaded with mixtures of $\mathrm{Sm} / \mathrm{Tb} / \mathrm{Tm}$. The monoliths have been excited at $\lambda_{\mathrm{ex}}=330 \mathrm{~nm}$.

\begin{tabular}{lll}
$\mathrm{Sm} / \mathrm{Tb} / \mathrm{Tm}$ ratio & $\mathrm{x}$ & $\mathrm{y}$ \\
\hline $8: 1: 91$ & 0.303 & 0.395 \\
$24: 1: 75$ & 0.349 & 0.405 \\
$19: 1: 80$ & 0.438 & 0.422 \\
$17: 1: 180$ & 0.326 & 0.349 \\
$29: 1: 170$ & 0.366 & 0.360 \\
$14.6: 0.3: 85.1$ & 0.363 & 0.327
\end{tabular}

Table 2a. Excited state lifetime data for monoliths loaded with mixtures of lanthanoid cations.

\section{Composition}

Emission Wavelength (nm)

\begin{tabular}{cccc}
$\mathbf{S m} / \mathbf{T b} / \mathbf{T m}$ & $\mathbf{5 4 6}$ & $\mathbf{5 9 8}$ & $\mathbf{6 4 6}$ \\
\hline $8: 1: 91$ & $321 \mu \mathrm{s}(27 \%)$ & $33 \mu \mathrm{s}(72 \%)$ & $32 \mu \mathrm{s}(87 \%)$ \\
& $847 \mu \mathrm{s}(73 \%)$ & $715 \mu \mathrm{s}(28 \%)$ & $638 \mu \mathrm{s}(23 \%)$ \\
$24: 1: 75$ & $274 \mu \mathrm{s}(30 \%)$ & $32 \mu \mathrm{s}(90 \%)$ & $31 \mu \mathrm{s}(94 \%)$ \\
& $852 \mu \mathrm{s}(70 \%)$ & $664 \mu \mathrm{s}(10 \%)$ & $592 \mu \mathrm{s}(6 \%)$
\end{tabular}




$\begin{array}{cccc}19: 1: 80 & 323 \mu \mathrm{s}(30 \%) & 31 \mu \mathrm{s}(91 \%) & 31 \mu \mathrm{s}(95 \%) \\ & 881 \mu \mathrm{s}(70 \%) & 650 \mu \mathrm{s}(9 \%) & 606 \mu \mathrm{s}(5 \%) \\ 17: 1: 180 & 280 \mu \mathrm{s}(31 \%) & 32 \mu \mathrm{s}(93 \%) & 32 \mu \mathrm{s}(97 \%) \\ & 853 \mu \mathrm{s}(69 \%) & 663 \mu \mathrm{s}(7 \%) & 636 \mu \mathrm{s}(3 \%) \\ 29: 1: 170 & 253 \mu \mathrm{s}(28 \%) & 32 \mu \mathrm{s}(93 \%) & 32 \mu \mathrm{s}(97 \%) \\ & 842 \mu \mathrm{s}(72 \%) & 668 \mu \mathrm{s}(7 \%) & 554 \mu \mathrm{s}(3 \%) \\ 14.6: 0.3: 85.1 & 252 \mu \mathrm{s}(27 \%) & 32 \mu \mathrm{s}(94 \%) & 32 \mu \mathrm{s}(92 \%) \\ & 833 \mu \mathrm{s}(73 \%) & 638 \mu \mathrm{s}(6 \%) & 633 \mu \mathrm{s}(8 \%)\end{array}$

Table 2b. Excited state lifetime data for monoliths loaded with $\mathrm{Tb}$ or $\mathrm{Sm}$.

Monolith Loading

$$
\mathrm{Tb}
$$

$\mathrm{Sm}$
Excited State Lifetime $(\tau)$

$$
326 \mu \mathrm{s}(30 \%) \text { and } 858(70 \%) \mu \mathrm{s} \text { at } \lambda_{\mathrm{em}}=548 \mathrm{~nm}
$$$$
17 \mu \mathrm{s}(30 \%) \text { and } 31 \mu \mathrm{s}(70 \%) \text { at } \lambda_{\mathrm{em}}=646 \mathrm{~nm}
$$

The emitted color of the loaded PMMA monolith can be finely tuned by varying the excitation wavelength (Figure 7). This color tuning as a function of the excitation wavelength is also clearly discernible from the analysis of the emission profiles, where the intensity of the blue band sequentially increases on gradually shifting $\lambda_{\mathrm{ex}}$ from 280 to $350 \mathrm{~nm}$. The effect can be understood by considering that the excitation spectrum of the blue component originating from the matrix has its maximum at $380 \mathrm{~nm}$, whereas the maximum of the excitation spectrum for the $\mathbf{1 H}$ ligand is centred around $330 \mathrm{~nm}$, as shown in Figure 8. The band around $330 \mathrm{~nm}$ in the excitation profile measured at $\lambda_{\mathrm{em}}=494,546,598$, and 646 $\mathrm{nm}$ are typical of the calix[4]arene ligand as expected for the sensitisation process. Moreover, the lower energy band in the excitation profile, visible at $\lambda_{\mathrm{em}}=494$, originates from the blue emission of the matrix. Again, this can be easily verified on comparing the excitation and emission profiles for the white- 
emitting materials with monoliths loaded exclusively with Sm, Tb, Tm or Gd (Figure 5).
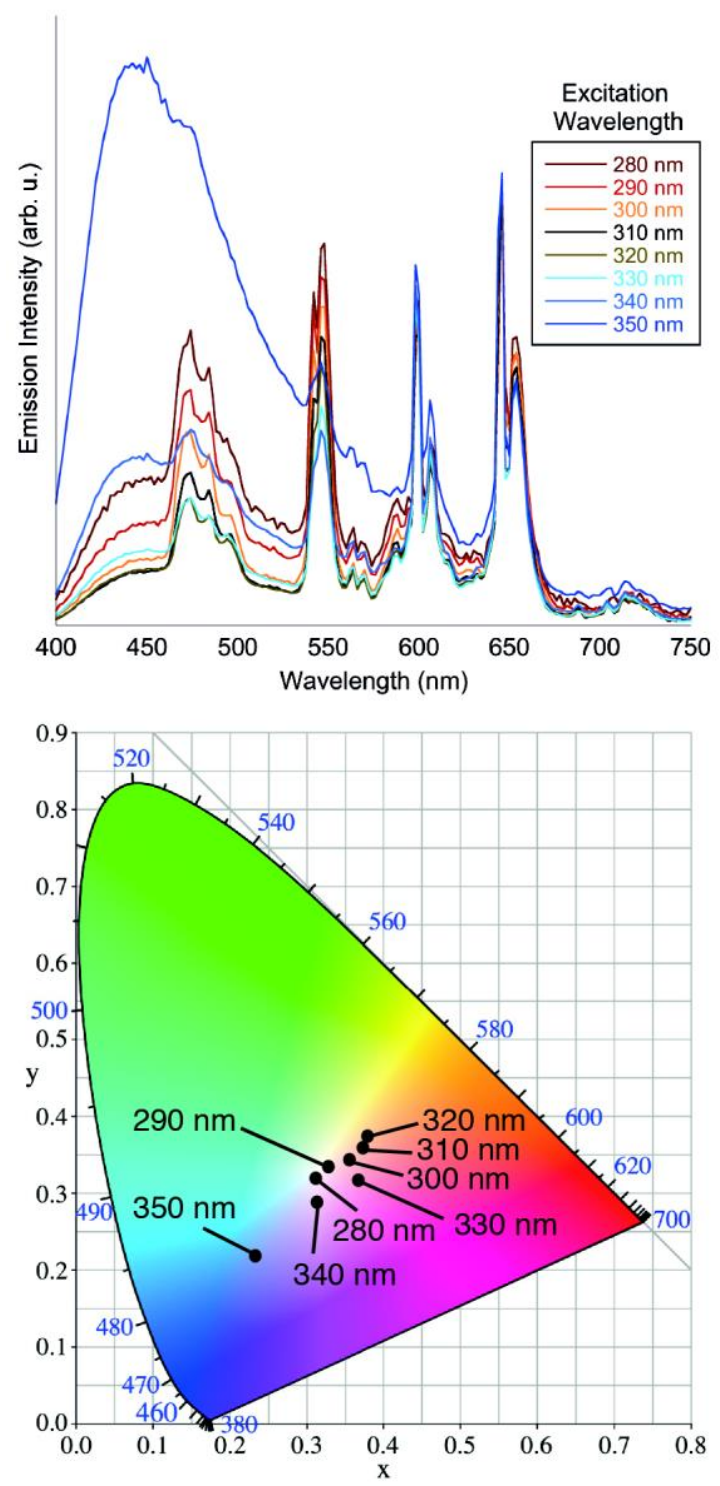

Figure 7. Wavelength dependent variation of the emission profile for a monolith loaded with a 29:1:170 $\mathrm{Sm} / \mathrm{Tb} / \mathrm{Tm}$ mixture (top); CIE-xy coordinates for the emission profile as a function of the excitation wavelength (bottom). 


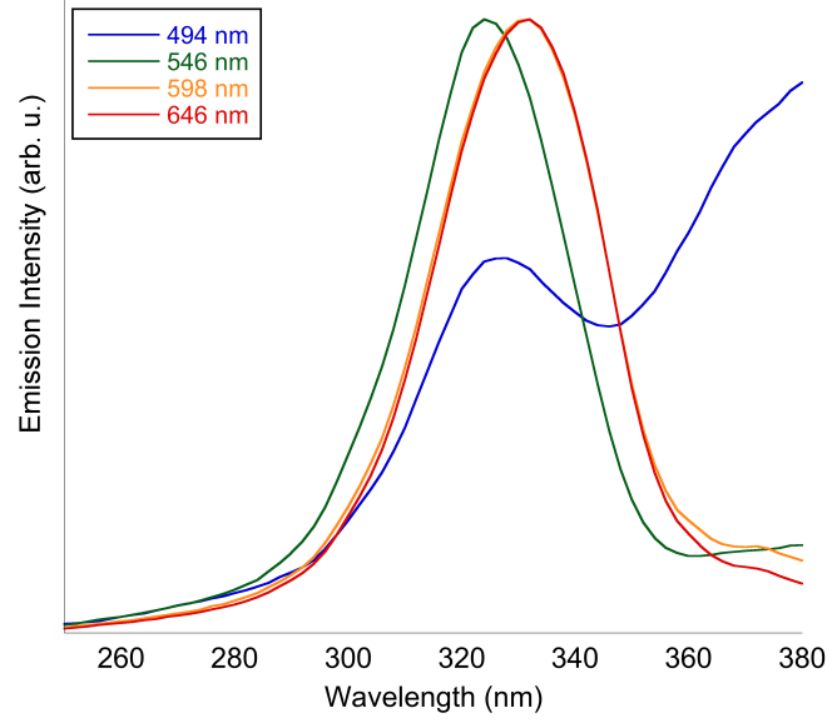

Figure 8. Excitation spectra of the white emitting polymer measured at different emission wavelengths (defined in the inset). The monolith consists of $0.2 \%$ molar equivalents of $\mathbf{1 H}$ and was loaded with a mixture of $\mathrm{Sm} / \mathrm{Tb} / \mathrm{Tm}$ with a molar ratio of 29:1:170.

Since the main contributor to the blue color in the emission spectrum at $\lambda_{\mathrm{ex}}=330 \mathrm{~nm}$ seems to belong to a radiative decay from the ${ }^{3}\left(\pi \pi^{*}\right)$ excited state of the calix[4]arene, the use of Tm in the mixture of lanthanoid elements is not necessary. In fact, white emissive materials could be obtained by loading the monoliths with a mixture of $\mathrm{Sm}, \mathrm{Tb}$, and $\mathrm{Gd}$ in a 32:1:7 molar ratio, respectively (Figure 9). The strong spin-orbit coupling of $\mathrm{Gd}$ ensures maximized emission from the ${ }^{3}\left(\pi \pi^{*}\right)$ excited state. ${ }^{24,}{ }^{25}$ Given the difference in price between precursors of Tm and $\mathrm{Gd}$ (about 20-25 times higher for $\mathrm{Tm}_{2} \mathrm{O}_{3}$ with respect to $\left.\mathrm{Gd}_{2} \mathrm{O}_{3}\right),{ }^{26}$ this substitution would be advantageous in any application of these materials. Moreover, the oxygen-sensitive nature of the emission of the Gd complex with $\mathbf{1 H}$ could be exploited for sensing purposes, by detecting the quenching the excited state in the presence of $\mathrm{O}_{2}$, an aspect that is currently under investigation in our laboratories. 

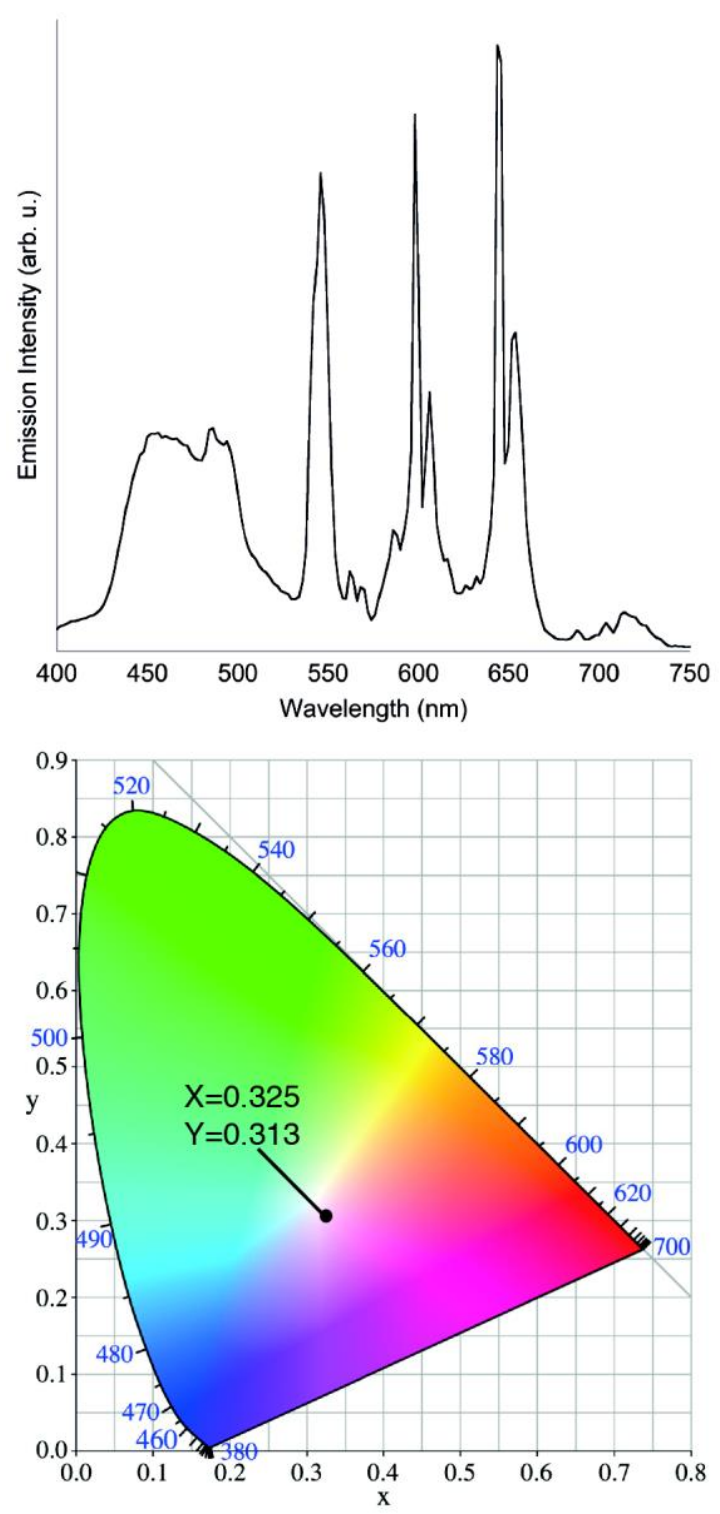

Figure 9. Emission profile of a monolith loaded with a mixture of 32:1:7 Sm/Tb/Gd (top); CIE-xy coordinates for the emission profile (bottom).

Reversibility of the lanthanoid loading: color switching

Remarkably, the composition of the hybrid materials, in terms of lanthanoid content, can be reversibly changed. As a demonstration, a white-emitting monolith of composition $\mathrm{Sm} / \mathrm{Tb} / \mathrm{Tm}$ 17:1:180, whose emission spectrum in shown in Figure 5, was immersed in a $10^{-3} \mathrm{M}$ solution of $\left[\mathrm{Tb}\left(\mathrm{NO}_{3}\right)_{3}(\mathrm{DMSO})_{\mathrm{n}}\right]$ or $\left[\mathrm{Sm}\left(\mathrm{NO}_{3}\right)_{3}(\mathrm{DMSO})_{\mathrm{n}}\right]$ in 1:1 dichloromethane/ethanol for 24 hours. After deswelling, green or orange emission was observed, respectively, at $\lambda_{\mathrm{ex}}=330 \mathrm{~nm}$ (Figure 10). On comparing the emission profiles before and after the exchange, it is evident that the $\mathrm{Tb}$ or $\mathrm{Sm}$ ions have predominantly filled the coordination sites of the cross-linking $\mathbf{1 H}$ ligands. The possibility of recycling the hybrid materials by switching their photophysical properties with a simple swelling process makes this method- 
ology unique, as to the best of our knowledge, no examples have been reported to date of hybrid materials with a composition that can be reversibly changed to tune or alter the functional (in this case emissive) properties. It should be also noted that no changes in the photophysical properties of the materials were detected if the polymers were swollen in just solvents, indicating a negligible tendency of the lanthanoid ions to leave a vacant calixarene coordination site in the washing phases.

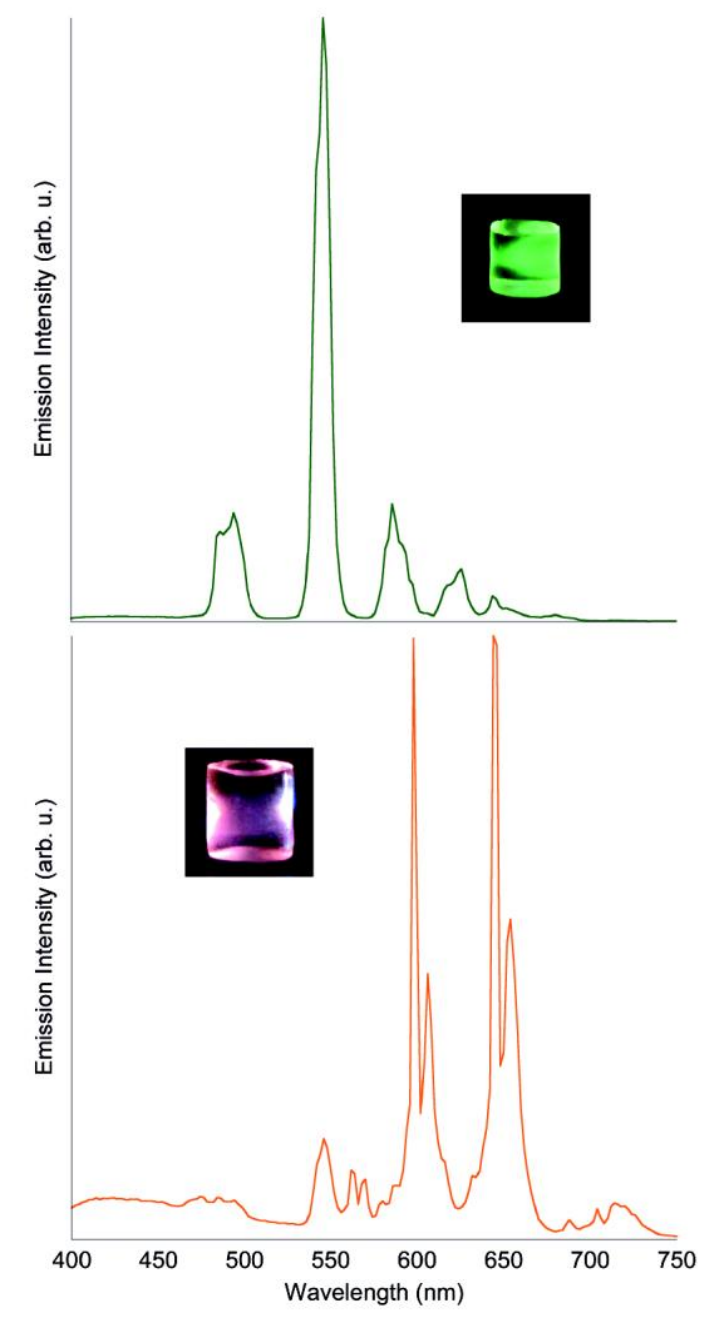

Figure 10. Emission spectra from a white emissive polymer after immersion in a solution of $\mathrm{Tb}$ (top) or Sm (bottom).

\section{Conclusions}

In conclusion, this work demonstrates that blank hybrid materials composed of PMMA crosslinked by allyl-functionalized calix[4]arene ligands can be readily loaded with lanthanoid ions, via a swelling/de-swelling methodology, and made luminescent. This procedure avoids loss of transparency due to phase segregation between the organic matrix and the lanthanoid coordination complex. The results highlight that mixtures of lanthanoid ions can be readily inserted within the material so that the emission properties originate from uniform mixing of the emission profiles of the single lanthanoids. 
While this has been demonstrated here to achieve balanced white light emission by combining red Sm, green $\mathrm{Tb}$, and blue $\mathrm{Tm} / \mathrm{Gd}$ complexes, the methodology has the potential to be easily extended to the preparation of materials emitting a large range of colors. In fact, according to color mixing theory, most of the colors can be obtained by an appropriate combination of three primary colors in the red, green and blue region of the visible spectrum. More importantly, the functional material can be recycled as needed to change the light emitted, by switching to a new lanthanoid combination using the same swelling/deswelling procedure. While $1 \mathrm{H}$ was originally designed to leave a vacant site in the coordination sphere for potential sensing applications, it is clearly possible to greatly enhance the photophysical and cation binding properties of the receptor, building on elegant examples from the literature. ${ }^{27-33}$ We are also investigating a diverse range of transparent polymeric hosts, so that this methodology can become a generic and facile procedure in the preparation of a large variety of luminescent and recyclable hybrid materials with color-switching properties.

Experimental Section

General All the reagents and solvents were obtained from Sigma Aldrich and used as received without any further purification, unless otherwise specified. Calixarene $1 \mathrm{H}^{20}$ and DMSO-solvated lanthanoid nitrate salts, $\left[\mathrm{Ln}\left(\mathrm{NO}_{3}\right)_{3}(\mathrm{DMSO})_{\mathrm{n}}\right],{ }^{34}$ were synthesized according to literature methods. Methyl methacrylate was distilled before use.

Photophysical measurements. Steady-state emission spectra were recorded on an Edinburgh FLSP920 spectrofluorimeter equipped with a $450 \mathrm{~W}$ Xenon arc lamp, single excitation and emission monochromators and a peltier cooled Hamamatsu R928P photomultiplier tube (185-850 nm). Emission and excitation spectra were corrected for source intensity (lamp and grating) and emission spectral response (detector and grating) by calibration curve supplied with the instrument. Excited state lifetimes $(\tau)$ were determined with the single photon counting technique (TCSPC) by means of the same Edinburgh FLSP920 spectrometer using pulsed picosecond LEDs (EPLED 295 or EPLED 360, FHWM <800 ps) with repetition rates between $10 \mathrm{kHz}$ and $1 \mathrm{MHz}$ as the excitation source and the above-mentioned R928P PMT as detector. The goodness of fit was assessed by minimizing the reduced chi squared function $\left(\chi^{2}\right)$ and visual inspection of the weighted residuals.

Preparation of the blank PMMA monoliths. $1 \mathrm{H}(0.0166 \mathrm{~g}, 0.018 \mathrm{mmol})$ and ethylene glycol dimethacrylate $(0.0056 \mathrm{~g}, 0.028 \mathrm{mmol})$ were dissolved in $1 \mathrm{~mL}$ aliquots of a lauroyl peroxide $(0.0125 \mathrm{~g}$, 
$0.031 \mathrm{mmol})$ in methyl methacrylate $(25.00 \mathrm{~g}, 250 \mathrm{mmol})$ solution. The reaction mixture was heated to $80^{\circ} \mathrm{C}$ for 3 minutes before being quenched in an ice-water bath. Polymerisation was carried out at $60^{\circ} \mathrm{C}$ for $24 \mathrm{hrs}$, then $70^{\circ} \mathrm{C}$ for $12-36 \mathrm{hrs}$. Finally a post-polymerisation step was carried out at $100^{\circ} \mathrm{C}$ for $1 \mathrm{hr}$.

Loading of blank monoliths with lanthanoid cations. $10^{-3} \mathrm{M}$ stock solutions of $\left[\mathrm{Ln}\left(\mathrm{NO}_{3}\right)_{3}(\mathrm{DMSO})_{\mathrm{n}}\right]$ salts in dichloromethane:ethanol (1:1), where $\mathrm{Ln}=\mathrm{Sm}, \mathrm{Tb}, \mathrm{Tm}$ and $\mathrm{Gd}$, were prepared. Polymer monoliths were loaded with lanthanoid cations by swelling in $4 \mathrm{~mL}$ of solution prepared from the stock solutions, according to the desired ratio of lanthanoid cations. Polymers were swollen for 48 hrs before deswelling at atmospheric pressure, followed by subsequent swelling with $4 \mathrm{~mL}$ of dichloromethane:ethanol (1:1) to remove any unbound lanthanoid cations from the polymer matrix. Monoliths were eventually de-swollen back to their original dimensions.

Recycling of luminescent monoliths. White-emitting monoliths of composition $\mathrm{Sm} / \mathrm{Tb} / \mathrm{Tm}$ 29:1:170 were immersed in a $4 \mathrm{~mL}$ solution of $\left[\mathrm{Ln}\left(\mathrm{NO}_{3}\right)_{3}(\mathrm{DMSO})_{\mathrm{n}}\right]$ in a 1:1 dichloromethane:ethanol mixture ( $\mathrm{Ln}$ $=\mathrm{Tb}$ or $\mathrm{Sm})$. After 24 hours, the swollen monoliths were removed from the solution and any excess lanthanoid cation was removed as described above.

\section{Acknowledgements}

The Australian Research Council (ARC) is acknowledged for funding support. M.M. wishes to thank the ARC for the APD Fellowship (DP0985481). Mr. Daniel d'Alessio is kindly acknowledged for help with the pictures of the luminescent monoliths. Dr. Evan G. Moore, University of Queensland, is kindly acknowledged for help with the calculation of the CIE-xy coordinates.

\section{References}

1. K. Binnemans, Chem. Rev., 2009, 109, 4283.

2. P. Gomez-Romero, Adv. Mater., 2001, 13, 163.

3. U. Schubert, Macromol. Symp., 2008, 267, 1.

4. K. Kuriki, Y. Koike and Y. Okamoto, Chem. Rev., 2002, 102, 2347.

5. J. M. Stanley and B. J. Holliday, Coord. Chem. Rev., 2012, 256, 1520.

6. M. Cavallini, J. Gomez-Segura, D. Ruiz-Molina, M. Massi, C. Albonetti, C. Rovira, J. Veciana and F. Biscarini, Angew. Chem. Int. Ed., 2005, 44, 888. 
7. J. C. G. Bünzli, Chem. Rev., 2010, 110, 2729.

8. A.-S. Chauvin, J. C. G. Bunzli, F. Bochud, R. Scopelliti and P. Froidevaux, Chem. Eur. J., 2006, 12, 6852 .

9. A. L. Jenkins, O. M. Uy and G. M. Murray, Anal. Commun., 1997, 34, 221.

10. T. H. Tran, M. M. Lezhnina and U. Kynast, J. Mater. Chem., 2011, 21, 12819.

11. J. C. G. Bünzli, Accounts Chem Res, 2006, 39, 53.

12. A. de Bettencourt-Dias, Dalton Trans., 2007, 2229.

13. Z. Sun, F. Bai, H. Wu, D. M. Boye and H. Fan, Chem. Mater, 2012, 27, 3415.

14. C.-j. Zhao, J.-1. Cai, R.-y. Li, S.-1. Tie, X. Wan and J.-y. Shen, J. Non-Cryst. Solids, 2012, 358, 604.

15. M.-S. Wang, S.-P. Guo, Y. Li, L.-Z. Cai, J.-P. Zou, G. Xu, W.-W. Zhou, F.-K. Zheng and G.-C. Guo, J. Am. Chem. Soc., 2009, 131, 13572.

16. L. Wu, Y. Zhang, M. Gui, P. Lu, L. Zhao, S. Tian, Y. Kong and J. Xu, J. Mater. Chem., 2012, 22, 6463 .

17. S. Dang, J.-H. Zhang and Z.-M. Sun, J. Mater. Chem., 2012, 22, 8868.

18. Y.-A. Li, S.-K. Ren, Q.-K. Liu, J.-P. Ma, X. Chen, H. Zhu and Y.-B. Dong, Inorg. Chem., 2012, 51, 9629..

19. S. H. Li, J. Qin, A. Fornara, M. Toprak, M. Muhammed and D. K. Kim, Nanotechnology, 2009, 20, 1 .

20. C. R. Driscoll, B. L. Reid, M. J. McIldowie, S. Muzzioli, G. L. Nealon, B. W. Skelton, S. Stagni, D. H. Brown, M. Massi and M. I. Ogden, Chem. Commun., 2011, 47, 3876.

21. P. C. Andrews, D. H. Brown, B. H. Fraser, N. T. Gorham, P. C. Junk, M. Massi, T. G. St Pierre, B. W. Skelton and R. C. Woodward, Dalton Trans., 2010, 39, 11227.

22. D. D'alessio, S. Muzzioli, B. W. Skelton, S. Stagni, M. Massi and M. I. Ogden, Dalton Trans., 2012, 41, 4736.

23. M. Latva, H. Takalo, V. M. Mukkala, C. Matachescu, J. C. RodriguezUbis and J. Kankare, J. Luminesc., 1997, 75, 149.

24. M. Hilder, M. Lezhnina, M. L. Cole, C. M. Forsyth, P. C. Junk and U. H. Kynast, J. Photochem. Photobiol., 2011, 217, 76.

25. M. Hilder, P. C. Junk, U. H. Kynast and M. M. Lezhnina, J. Photochem. Photobiol. A 2009, 202, 10.

26. Sigma-Aldrich, 2012 catalogue.

27. A. Casnati, C. Fischer, M. Guardigli, A. Isernia, I. Manet, N. Sabbatini and R. Ungaro, J. Chem. Soc. Perkin Trans. 2, 1996, 395.

28. C. Fischer, G. Sarti, A. Casnati, B. Carrettoni, I. Manet, R. Schuurman, M. Guardigli, N. Sabbatini and R. Ungaro, Chem. Eur. J., 2000, 6, 1026.

29. L. Prodi, S. Pivari, F. Bolletta, M. Hissler and R. Ziessel, Euro. J. Inorg. Chem., 1998, 1959. 
30. D. M. Rudkevich, W. Verboom, E. Vandertol, C. J. Vanstaveren, F. M. Kaspersen, J. W. Verhoeven and D. N. Reinhoudt, J. Chem. Soc. Perkin Trans. 2, 1995, 131.

31. F. J. Steemers, W. Verboom, D. N. Reinhoudt, E. B. Vandertol and J. W. Verhoeven, J. Am. Chem. Soc., 1995, 117, 9408.

32. G. Ulrich, R. Ziessel, I. Manet, M. Guardigli, N. Sabbatini, F. Fraternali and G. Wipff, Chem. Eur. J., 1997, 3, 1815.

33. I. Oueslati, R. A. S. Ferreira, L. D. Carlos, C. Baleizao, M. N. Berberan-Santos, B. de Castro, J. Vicens and U. Pischel, Inorg. Chem., 2006, 45, 2652.

34. S. K. Ramalingam and S. Soundararajan, J. Inorg. Nucl. Chem., 1967, 29, 1763. 